DAMTP-2011-100

\title{
Monopole Planets and Galaxies
}

\author{
N. S. Manton 1 \\ Department of Applied Mathematics and Theoretical Physics, \\ University of Cambridge, \\ Wilberforce Road, Cambridge CB3 0WA, U.K.
}

\begin{abstract}
Spherical clusters of $S U(2)$ BPS monopoles are investigated here. A large class of monopole solutions is found using an abelian approximation, where the clusters are spherically symmetric, although exact solutions cannot have this symmetry precisely. Monopole clusters generalise the Bolognesi magnetic bag solution of the same charge, but they are always larger. Selected density profiles give structures analogous to planets of uniform density, and galaxies with a density decaying as the inverse square of the distance from the centre. The Bolognesi bag itself has features analogous to a black hole, and this analogy between monopole clusters and astrophysical objects with or without black holes in their central region is developed further. It is also shown that certain exact, platonic monopoles of small charge have sizes and other features consistent with what is expected for magnetic bags.
\end{abstract}

Keywords: BPS Monopoles, Magnetic Bags, Planets, Galaxies, Black Holes PACS: 14.80.Hv, 11.27.+d, 96.12.Fe, 98.62.Ck, 04.70.Bw

\footnotetext{
${ }^{1}$ email: N.S.Manton@damtp.cam.ac.uk
} 


\section{Introduction}

SU(2) Yang-Mills gauge theory with an adjoint scalar Higgs field is a classic example of a nonlinear field theory in three space dimensions with smooth, topological soliton solutions. The solitons are magnetic monopoles [1, 2, 3]. $\mathrm{SU}(2)$ magnetic monopoles were first discovered by 't Hooft and Polyakov, but the special case pinpointed by Bogomolny, Prasad and Sommerfield (BPS) has received particular attention from physicists and mathematicians. Static BPS monopoles obey a first order partial differential equation known as the Bogomolny equation, $\mathbf{B}=\mathbf{D} \Phi$, where $\mathbf{B}$ is the Yang-Mills magnetic field and $\mathbf{D} \Phi$ is the covariant gradient of the Higgs field $\Phi$. In addition, there is the boundary condition that at spatial infinity, $\Phi$ is of unit magnitude; in other words, $\Phi$ has unit vacuum expectation value. This Bogomolny equation is a three-dimensional analogue of the four-dimensional self-dual Yang-Mills equation, whose solutions are instantons. It is not easy to solve explicitly.

Monopole solutions are characterised by a topological charge $N$, a positive integer that can be identified with the number of basic monopoles, so solutions with charge $N$ are $N$-monopole configurations. The basic monopole of unit charge is spherically symmetric and has core size 1. Outside the core, the fields abelianize and resemble those of a Dirac monopole, with a magnetic Coulomb tail, and in addition a long-range Higgs field tail.

$N$-monopole solutions of the Bogomolny equation are plentiful [4], even after the gauge freedom is quotiented out. The magnetic and scalar forces between static BPS monopoles cancel [5], so there is great freedom in where the constituent monopoles can be located, and it has been rigorously established that there is a connected $4 N$-dimensional moduli space of $N$-monopole solutions. There is a deep mathematical theory, combining ideas of integrable systems, holomorphic bundles, Nahm data and spectral curves, and a minitwistor space, giving insights into the Bogomolny equation and its monopole solutions [1, 2]. The monopoles of charge $N$ are in 1-1 correspondence with (based) rational maps of degree $N$, from $S^{2}$ to $S^{2}$, known as Jarvis maps in this context [6]. By considering Jarvis maps, it becomes clear that no BPS monopole of charge greater than 1 can be exactly spherically symmetric, so a cluster of monopoles can be at most approximately spherically symmetric.

The 1-monopole solution has a simple closed form, and much is known about 2-monopole solutions [1]. A 2-monopole solution has just one interesting parameter, the spatial separation of the pair. However, the the field values and energy density are complicated functions of spatial position and monopole separation. For charges higher than 2, only certain specially symmetric monopoles are known precisely [2]. Even here, what is known are the 
spectral curves and the rational maps associated with the monopole. The fields and energy distributions need to be calculated numerically. Examples are a few monopoles with platonic symmetry, with charges $N \leq 11$. There is also a family of $N$-monopole solutions, for all $N$, which are regular polygons of $N$ unit charge monopoles [7]. When the radius of the regular $N$-gon solution is minimised, the $N$ monopoles merge, and the energy density is concentrated in a circular ring, or torus [8, 9, 10]. The platonic monopoles, and the family of toroidal monopoles for all $N>1$, are among the most compact monopole solutions.

If all $N$ monopoles are very well separated from each other, then the solution is a superposition of $N$ particle-like objects, each with little distortion. The $4 N$ parameters are the $N$ positions of the unit charge monopoles $(3 N$ parameters) and $N$ phases which are required to define the gluing of their fields into space. However, there is little insight so far into the spatial structure of a generic $N$-monopole solution, where the monopoles form a relatively compact cluster. Monopoles cannot be very close together, as they get larger as they approach each other, so it is not clear how big such a cluster might be.

The purpose of this paper is to shed some light on monopole clusters of large charge, and our method is to extend the analysis that was used by Bolognesi to discover and investigate a further class of BPS monopole solutions called magnetic bags [11].

The existence of magnetic bags has not been rigorously proved, although it is likely they exist for all large $N$. The bags are approximately spherical monopole solutions of charge $N$, with the nonabelian structure [12] concentrated on a thin spherical surface of radius approximately $N$. Because of the surface structure, the fields are not exactly spherically symmetric. Bags can be distorted away from spherical, but we shall focus on those that are not. What is known precisely about magnetic bags are the abelianized gauge and Higgs fields that they produce. These resemble the electric field and the electrostatic potential arising from a charged conductor.

The spherical magnetic bags are especially interesting, because, for each $N$, they appear to be the most compact $N$-monopole solutions that there are. We shall present new evidence for this in this paper. Our main purpose, however, is to generalise the construction of the magnetic bags to a much larger class of solutions that can be interpreted as spherical clusters of monopoles. Dilute clusters of $N$ unit charge monopoles are very large, because the size of each constituent monopole depends on the Higgs field in the neighbourhood. If the background abelianized Higgs field is $\phi$, then locally a monopole has radius $1 / \phi$. Our calculations below will give lower bounds on the size of monopole clusters based on this basic property of the 
constituent monopoles. In a spherical cluster, $\phi$ is smallest near the centre, so here the monopoles are largest.

In Section 2 we shall review the properties of the Bolognesi magnetic bag, and then in Section 3 present a multi-layer generalisation of the magnetic bag, assuming spherical symmetry for simplicity. We shall establish an important constraint on the radial distribution of charge in such a structure. Various inequalities follow from this, and one of our conclusions will be that no multi-layer solution packs all $N$ monopoles into a smaller volume than the Bolognesi bag. In Section 4 we give a smoothed-out description of a multi-layer magnetic bag, which now becomes a cluster of monopoles with a quasi-smooth charge density. The density is a rather arbitrary function of distance from the centre, subject to one constraint. We discuss whether a Bolognesi type of bag persists in the inner part of the cluster.

In Sections 5 and 6 we present examples of monopole clusters that have density profiles typical of two types of astrophysical object - the first is a planet, with a constant density inside and zero density outside; the second is a spherical galaxy, with a density falling with the inverse square of the distance from the centre [13]. We give these two types of cluster the names monopole planet and monopole galaxy. There are some analogies between monopole clusters subject to Yang-Mills-Higgs forces, and astrophysical objects subject to gravitational forces, including black holes. We shall not simultaneously consider both types of force, although Bolognesi has recently considered gravitating magnetic bags [14].

In Section 7, which depends on more technical material about exact monopole solutions, we show, by calculating spectral radii, that the known monopoles with platonic symmetries are examples of approximately spherical magnetic bags, of small charge. We also show that the toroidal monopole of charge $N$ is not as compact as the spherical magnetic bag of charge $N$.

In Section 8 we present our conclusions, and summarise the analogy that appears to exist between monopole clusters and astrophysical objects, which extends the analogy between magnetic bags and black holes that has been noticed by Bolognesi [14]. 


\section{Magnetic Bags}

An interesting structure, discovered by Bolognesi and called a magnetic bag [11], is possible for a monopole with a large charge $N$. It is an abelian approximation to what is expected to be an exact solution of the $S U(2)$ Bogomolny equation $\mathbf{B}=\mathbf{D} \Phi$. The simplest version of the bag is spherically symmetric. The abelian fields are a scalar field $\phi$, representing the magnitude of the Higgs field $\Phi$, and a magnetic field $\mathbf{b}$, the projection of the $S U(2)$ magnetic field $\mathbf{B}$ onto the direction of $\Phi$ in the $S U(2)$ Lie algebra. They are related by $\mathbf{b}=\nabla \phi$, which is the abelianized Bogomolny equation. Away from the monopoles $\nabla \cdot \mathbf{b}=0$, so $\nabla^{2} \phi=0$; the monopoles are Coulombtype sources for $\mathbf{b}$. In the interior of the bag $\phi=0$, and in the exterior $\phi=1-N / r$. Correspondingly, $\mathbf{b}$ vanishes in the interior and $\mathbf{b}=N \mathbf{r} / r^{3}$ in the exterior. At the bag surface, separating interior from exterior, $\phi$ is required to be continuous, so the bag is at radius $R=N$. Note that $\nabla \phi$ and $\mathbf{b}$ are discontinuous across the bag, the discontinuity of the flux of $\mathbf{b}$ being $4 \pi N$. The interpretation is that the bag carries magnetic charge $N$, spread uniformly over the surface, and that the interior of the bag is a magnetic conductor, carrying no charge.

It is very plausible that there is an exact solution, approximated by the bag, which has abelianized fields as described above except in a thin spherical wall at radius $N$, where the fields are fully nonabelian. The bag wall is of thickness approximately $\sqrt{N}$, and is a slightly curved version of the flat monopole wall solution found by Ward [15]. That solution has a twodimensional lattice periodicity, which is why the bag cannot be exactly spherically symmetric. The spherical bag must not only curve the wall, but also introduce defects in the two-dimensional lattice structure. Nevertheless, the lattice structure probably survives locally. Lee and Weinberg have described in considerable detail some of the topological features of the nonabelian fields of the bag [12]. Nahm data associated with magnetic bags of large charge have been discussed by Harland [16].

Some evidence for the existence of bags comes from the platonic monopoles [7, 17, 18]. These are exact solutions of the Bogomolny equation of low charge which resemble bags, in that they are hollow platonic polyhedra, with energy and nonabelian field structures concentrated on their surfaces. So far, their radii have not been compared with the Bolognesi estimate $R=N$. Using the spectral curves of these monopoles, we calculate their radii in Section 7 here, and show that they are close to the Bolognesi value. As a definition of size we use the spectral radius [1].

Perhaps the most surprising property of a magnetic bag is its large size. 
Since the radius is $N$, its area is a multiple of $N^{2}$ and its volume a multiple of $N^{3}$. One might expect BPS monopoles to be a model of rather normal matter, where $N$ particles fit into a volume of order $N$, but this is not the case. The magnetic bag is much larger. Black holes have a similarly exotic size scaling. The radius of the horizon of a Schwarzschild black hole of mass $M$ is $2 M$ in natural units, and the black hole area is proportional to $M^{2}$. A magnetic bag is therefore, to some extent, a gauge theory analogue of a black hole.

It is worth recalling a few more properties of the magnetic bag, and some variants of the bag, pointed out by Bolognesi, and by Lee and Weinberg. First, the bag can be deformed from spherical, because of the large number of dimensions of the moduli space of $N$ monopoles. However, we will only consider spherical distributions of monopoles here. Next, there is a rather trivial rescaling of the Bolognesi bag if the vacuum expectation value of the Higgs field $\Phi$ is $v$, rather than the special value $v=1$. One now has $\phi=v-N / r$ outside the bag, and $\phi=0$ inside, so the bag radius is $R=N / v$. Its thickness is $\sqrt{N} / v$ [12]. The argument for this is that the bag area is $N^{2} / v^{2}$ (dropping constants of order 1 ), so the area per unit charge monopole is $N / v^{2}$. The characteristic length associated with a monopole in the bag surface (which can be thought of as the monopole separation within the surface) is therefore the square root of this, $\sqrt{N} / v$, and since it is the only length scale in the bag, the bag thickness must be the same. We will, in fact, always suppose that $v=1$ and impose the boundary condition that $\phi \rightarrow 1$ as $r \rightarrow \infty$. However, the bag solution with other values of $v$ will be locally relevant. Note that for any $v$ the bag thickness is $R / \sqrt{N}$, where $R$ is the radius and $N$ the charge.

Lastly, we recall that there is a variant bag of charge $N$ where $\phi$ does not vanish inside, but instead $\phi=c$ inside, with $0<c<1$. Outside, $\phi=1-N / r$ as usual, so the bag radius is $R=N /(1-c)$, making $\phi$ continuous across the bag surface. This bag radius is larger than the radius of the Bolognesi bag, and if $c$ is not very small, then the bag breaks up into constituent, unit charge monopoles. To estimate the critical value of $c$ where break-up occurs, note that since the Higgs field is $\phi=c$ in the bag vicinity, unit charge monopoles have a radius $1 / c$. The bag has broken up into constituents if the monopole separation within the bag, $\sqrt{N}$, is bigger than this. The critical value of $c$ is therefore $1 / \sqrt{N}$, which is much less than 1 if $N$ is large. The critical value of $c$ is reached when the bag has expanded to a radius approximately $N+\sqrt{N}$. If the bag is larger than this it should be regarded as a spherical layer of separated, unit charge monopoles, rather than as a Bolognesi bag. 


\section{Multi-layer Magnetic Bags}

Using the abelianized Bogomolny equation $\mathbf{b}=\nabla \phi$, it is straightforward to calculate the Higgs field $\phi$ and magnetic field $\mathbf{b}$ of a multi-layer structure of several magnetic bags with spherical symmetry. Let us suppose that there are bags with positive integer charges $\left\{N_{1}, N_{2}, \ldots, N_{K}\right\}$ on spheres of radii $\left\{R_{1}, R_{2}, \ldots, R_{K}\right\}$, where $0<R_{1}<R_{2}<\ldots<R_{K}$. The total charge is $N=N_{1}+N_{2}+\cdots+N_{K}$. Let $b$ denote the magnitude of the radial magnetic field b. $b(r)$ has discontinuities at each bag. Between $R_{k}$ and $R_{k+1}$,

$$
b(r)=\frac{N_{1}+N_{2}+\cdots+N_{k}}{r^{2}},
$$

the magnetic analogue of the Coulomb field due to the enclosed charge. Also $b(r)=0$ for $r<R_{1}$ and $b(r)=N / r^{2}$ for $r>R_{K}$. The Higgs field $\phi(r)$ satisfies

$$
\frac{d \phi}{d r}=b(r) .
$$

If we integrate out from $r=0$, and assume $\phi(0)=0$, then we find that $\phi(r)=0$ for $r<R_{1}$,

$$
\phi(r)=\frac{N_{1}}{R_{1}}+\frac{N_{2}}{R_{2}}+\cdots+\frac{N_{k}}{R_{k}}-\frac{N_{1}+N_{2}+\cdots+N_{k}}{r}
$$

between $R_{k}$ and $R_{k+1}$, and

$$
\phi(r)=\frac{N_{1}}{R_{1}}+\frac{N_{2}}{R_{2}}+\cdots+\frac{N_{K}}{R_{K}}-\frac{N}{r}
$$

for $r>R_{K}$. $\phi$ is continuous at each bag radius, but $d \phi / d r$ has a discontinuity.

The boundary condition $\phi \rightarrow 1$ as $r \rightarrow \infty$ requires that

$$
\frac{N_{1}}{R_{1}}+\frac{N_{2}}{R_{2}}+\cdots+\frac{N_{K}}{R_{K}}=1
$$

This is the key constraint on multi-layer bags and their distribution of charges. This constraint is the generalisation of the result that for a single Bolognesi bag, $R=N$. Various inequalities follow. Among them we have

$$
\frac{N_{k}}{R_{k}}<1,
$$

so each bag is larger than it would be if it had the same charge but were alone. More interesting is to consider the total charge $N$. Replacing each 
radius $R_{k}$ by $R_{1}$ we increase the sum on the left hand side of eq.(15) to a value greater than 1 . But the sum is now $N / R_{1}$, so $R_{1}<N$. Similarly, replacing $R_{k}$ by $R_{K}$, we find $R_{K}>N$. Thus, compared with a Bolognesi bag of charge $N$, the multi-layer structure has a smaller innermost bag, and a larger outermost bag. The structure as a whole is larger than the Bolognesi bag. Indeed, this is strong evidence that the smallest radius into which one can fit $N$ monopoles is the Bolognesi radius $R=N$.

A very similar solution with $\phi(r)=c$ for $r<R_{1}$ (with $0<c<1$ ) exists when

$$
\frac{N_{1}}{R_{1}}+\frac{N_{2}}{R_{2}}+\cdots+\frac{N_{K}}{R_{K}}=1-c .
$$

Here $R_{K}>N /(1-c)$, so the solution is larger than before.

Let us now consider the type of structure each bag layer has. For the rest of this section we assume $c=0$. The innermost layer is a bag of Bolognesi type. It has charge $N_{1}$ and radius $R_{1}$, the Higgs field is $\phi=0$ inside and $\phi(r)=N_{1} / R_{1}-N_{1} / r$ outside (as far as the second layer). This is just as expected for a Bolognesi bag of charge $N_{1}$ and effective vacuum expectation value $v=N_{1} / R_{1}$. The thickness of this bag is $\sqrt{N_{1}} / v=R_{1} / \sqrt{N_{1}}$, which is small compared to $R_{1}$ provided $N_{1}$ is large. The second layer needs to be further from the first than this thickness, for the multi-layer point of view to be justified.

Let us therefore assume that the separation $R_{2}-R_{1}$ is significantly larger than $R_{1} / \sqrt{N_{1}}$, but significantly less than $R_{1}$. Let us also assume that the charges in the first and second layer are similar. Within the second layer the monopole separation is $R_{2} / \sqrt{N_{2}}$, which is comparable to the separation within the first layer. Just inside the second layer the Higgs field is

$$
\phi=\frac{N_{1}}{R_{1}}-\frac{N_{1}}{R_{2}}=\frac{N_{1}\left(R_{2}-R_{1}\right)}{R_{1} R_{2}}
$$

which, by our assumptions, is considerably larger than both $\sqrt{N_{1}} / R_{2}$ and $\sqrt{N_{2}} / R_{2}$. We estimate the monopole size in the second layer as $1 / \phi=$ $R_{1} R_{2} /\left(N_{1}\left(R_{2}-R_{1}\right)\right)$, and this is considerably less than the monopole separation within the layer, $R_{2} / \sqrt{N_{2}}$. The second layer is therefore not a Bolognesi bag, but instead a spherical arrangement of well separated unit charge monopoles. Since $\phi$ increases as one moves out to subsequent layers, the characteristic size of a monopole decreases. On the other hand, the monopole separation within each layer increases as one moves out, provided the charges in all the layers are similar. It follows that all the outer layers consist of well separated monopoles, rather than a bag of Bolognesi type. The entire solution consists of an inner layer which is a Bolognesi bag, surrounded by 
a layered cluster of well separated monopoles. These monopoles are not of equal size. They are largest near the centre, where the Higgs field is small, and decrease in size further out, with monopoles in the outer layers having approximately unit size.

A multi-layer bag solution may have a Bolognesi type of bag as one of the intermediate layers. But this only happens if the distribution of charges is very uneven, with the constraint (5) satisfied by having one ratio $N_{k} / R_{k}$ very close to 1 , and all the others much less than 1 . The $k$ th layer is then a Bolognesi bag. It is still possible for one of the layers closer to the centre to be a Bolognesi bag too, but its charge must be very much smaller. We can verify this by considering a structure with just two layers, each with a large charge (so that the square root of the charge is small compared with the charge itself). If we fix their radii at $R_{1}$ and $R_{2}$ (with ratio of order 1 ), and satisfy the constraint

$$
\frac{N_{1}}{R_{1}}+\frac{N_{2}}{R_{2}}=1
$$

by setting $N_{1} / R_{1}=\varepsilon$ and $N_{2} / R_{2}=1-\varepsilon$ with $\varepsilon$ small, then the inner layer is a Bolognesi bag automatically, and the outer layer is one too, provided $\phi$ is sufficiently small at $r=R_{2}$. This occurs if $\varepsilon<1 / \sqrt{N_{2}}$. Since $N_{2}$ is approximately $R_{2}$, and $N_{1}=\varepsilon R_{1}<\varepsilon R_{2} \simeq \varepsilon N_{2}, N_{1}$ needs to be less than $\sqrt{N_{2}}$. As anticipated, the inner charge is much smaller than the outer charge.

Our main conclusion is that multi-layer bag structures are possible, but the layers are not generally of the Bolognesi bag type. Typically, the innermost layer is a Bolognesi bag, and it is surrounded by spherical layers of isolated unit charge monopoles. We shall next consider the continuum limit of these structures. There will be a spherically symmetric density of magnetic charge located between an inner and outer radius. At the inner radius we shall find there is often a Bolognesi bag, and outside this, a gas of isolated monopoles.

\section{A Smooth, Spherical Cluster of Monopoles}

For a spherical cluster of BPS monopoles, of very large charge, the continuum limit arises when the layers discussed above merge into a charge density that is a quasi-continuous function of the radius. It is convenient to work with the radial density $q(r)$, defined so that the charge in the shell between radius $r$ and radius $r+d r$ is $q(r) d r$. For monopoles satisfying the Bogomolny equation, 
$q(r) \geq 0$ everywhere. The charge $Q(R)$ interior to radius $R$ is

$$
Q(R)=\int_{0}^{R} q(r) d r
$$

and the magnetic field strength is $b(R)=Q(R) / R^{2}$. We shall assume that the total charge, $Q=\lim _{R \rightarrow \infty} Q(R)=N$, is finite.

The constraint (15) becomes, in the continuum limit,

$$
\int_{0}^{\infty} \frac{q(r)}{r} d r=1
$$

This is in the case that the Higgs field $\phi$ runs from 0 at the origin to 1 at infinity. More generally, if $\phi$ has a positive value $c$ at the origin,

$$
\int_{0}^{\infty} \frac{q(r)}{r} d r=1-c .
$$

These integrals automatically converge at infinity if the total charge is finite, but will fail to converge at the origin unless the density $q$ tends to zero sufficiently fast there. Having a finite, non-zero volume density of charge at the origin makes $q(r)$ proportional to $r^{2}$ for small $r$, and this is one way to ensure convergence. We shall also consider $q$ strictly vanishing inside an interior radius $R_{\text {in }}$. It also makes sense to assume that $q$ vanishes outside some outer radius $R_{\text {out }}$. This is because monopoles each have at least one unit of charge, so that a smoothly decaying charge density stretching to infinity is not possible.

With the outer radius assumed, the total charge is

$$
Q=\int_{0}^{R_{\mathrm{out}}} q(r) d r
$$

and the constraint (12) is

$$
\int_{0}^{R_{\text {out }}} \frac{q(r)}{r} d r=1-c
$$

Replacing $r$ by $R_{\text {out }}$ in the denominator gives the inequality $Q / R_{\text {out }}<1-c$ (assuming $q$ not entirely concentrated at $R_{\text {out }}$ ), so the ball that contains all the charge has radius larger than $Q /(1-c)$, the radius of the one-layer bag for the same total charge.

We will need the formula for the Higgs field $\phi$. By analogy with the multi-layer case, or by direct integration, we find

$$
\phi(R)=\int_{0}^{R} \frac{q(r)}{r} d r-\frac{Q(R)}{R}+c .
$$


This is analogous to the Newtonian gravitational potential for a spherical mass distribution, shifted so that $\phi=1$ at infinity. Using the constraint (14), we have the alternative expression

$$
\phi(R)=1-\int_{R}^{R_{\mathrm{out}}} \frac{q(r)}{r} d r-\frac{Q(R)}{R} .
$$

$\phi(R)$ obeys the spherically symmetric version of the Poisson equation

$$
\frac{d}{d R}\left(R^{2} \frac{d \phi}{d R}\right)=q(R)
$$

and $b(R)=d \phi / d R$ as before.

For generic densities $q(r)$, we anticipate that if $c=0$ a Bolognesi bag could occur at some inner radius, with a gas of isolated monopoles outside. For sufficiently non-uniform $q(r)$ there could be Bolognesi bags at different radii, as we saw with the multi-layer structures, but we will not seek these out again. In the next sections, we consider two specific densities, corresponding to what we think of as a monopole planet and a monopole galaxy. The monopole galaxy has a Bolognesi bag at its inner radius.

\section{Monopole Planets}

A (small) planet is a spherical body where gravity plays a limited role. If the material is uniform, the density is uniform, dropping suddenly to zero at the planet's outer radius. By a monopole planet, we mean a spherical cluster of monopoles with a radial density $q(r)=4 \pi \rho r^{2}$ between $r=0$ and $r=R_{\text {out }}$, with $\rho$, the charge density per unit volume, a positive constant. The density vanishes outside. As the volume per unit charge is $\rho^{-1}$ we estimate the nearest neighbour monopole separation as $\rho^{-1 / 3}$. Here, and in the rest of this and the next section, factors of order 1 are often ignored.

The total charge of the planet is

$$
Q=\frac{4 \pi}{3} \rho R_{\text {out }}^{3}
$$

and the constraint (14) simplifies here to

$$
2 \pi \rho R_{\text {out }}^{2}=1-c .
$$


Eliminating $\rho$ in favour of $Q$ we find

$$
R_{\text {out }}=\frac{3}{2(1-c)} Q \text {. }
$$

This is always greater than the Bolognesi radius $R=Q$.

One might imagine that for given charge $Q, R_{\text {out }}$ is smallest when $c=0$, but this choice of $c$ is not consistent. Close to the origin $\phi$ would be so small that the monopole sizes would exceed their separation. To restore consistency we would need to dilute the monopole density near the origin, creating a hole. We do not want to do this here, but will do so in the next section. Instead, let us keep the density uniform, but find the minimal consistent value of $c$. This is the value $c=\rho^{1 / 3}$, for which the monopole size close to the origin, which is $1 / c$, matches the monopole separation $\rho^{-1 / 3}$. Again we can eliminate $\rho$ in favour of $Q$. We find that $c=O\left(Q^{-2 / 3}\right)$ if $Q$ is large. So the monopoles close to the centre of the planet have size of order $Q^{2 / 3}$ (and hence volume $Q^{2}$ ), much larger than the monopoles near the outside, which have unit size. From (20) it follows that this densest possible monopole planet has size $R_{\text {out }}=3 Q / 2+O\left(Q^{1 / 3}\right)$. The Higgs field $\phi$ is small near the centre but not zero, so there is no magnetic bag in the centre. A less dense monopole planet of the same charge arises if $c$ has a larger value.

\section{Monopole Galaxies}

The remarkable feature of many spherical and almost spherical galaxies is that their density is highly non-uniform, decaying as the inverse square of distance from the centre [13]. This density profile is called a singular isothermal sphere. We shall investigate here the structure of a BPS monopole of large charge with such a density profile. If the charge density per unit volume decays as $r^{-2}$ then the radial density $q(r)$ is a constant, $q_{0}$. Let us suppose that $q(r)=q_{0}$ in the radial range $R_{\text {in }} \leq r \leq R_{\text {out }}$ and vanishes outside this range. The total charge $Q$ is $q_{0}\left(R_{\text {out }}-R_{\text {in }}\right)$. We want a finite charge, so $R_{\text {out }}$ is finite. If $R_{\text {in }} \ll R_{\text {out }}$ then the total charge is approximately $q_{0} R_{\text {out }}$.

The usual constraint on the radial density becomes

$$
\int_{R_{\mathrm{in}}}^{R_{\mathrm{out}}} \frac{q_{0}}{r} d r=1-c,
$$

that is,

$$
q_{0}\left(\log R_{\text {out }}-\log R_{\text {in }}\right)=1-c .
$$


This constraint forces $R_{\text {in }}$ to have a positive value. Having an inner cut-off at a positive radius is the same as in galaxy modelling, and avoids a singular density at the origin. The interesting case is where $c=0$. The Higgs field $\phi$ then vanishes for $r \leq R_{\text {in }}$, and we anticipate that there can be a Bolognesi bag in the neighbourhood of $R_{\text {in }}$, surrounded by a more dilute gas of well separated monopoles. Note that $q_{0}$ is not very different from 1 for a large range of ratios $R_{\text {out }} / R_{\text {in }}$.

We need the Higgs field $\phi$ between $R_{\text {in }}$ and $R_{\text {out }}$. The expression (16) reduces to

$$
\phi(R)=1-q_{0}\left(\log R_{\text {out }}-\log R\right)-q_{0}\left(1-\frac{R_{\text {in }}}{R}\right) .
$$

For $R$ near $R_{\text {out }}$ this has the characteristic logarithmic dependence on $R$ familiar from the Newtonian gravitational potential in galaxy models. Near $R_{\text {in }}$ there is a quadratic dependence on $R-R_{\text {in }}$, and the slope of $\phi$ (to leading order) is

$$
\frac{d \phi}{d R}=\frac{q_{0}}{R_{\mathrm{in}}^{2}}\left(R-R_{\mathrm{in}}\right)
$$

Now recall that for the multi-layer structures discussed in section 3, with $\phi=0$ in the interior, the innermost layer is usually a Bolognesi bag. The charge $N_{1}$ and radius $R_{1}$ are arbitrary, but they are related to the slope of $\phi$ just outside the bag, which is $N_{1} / R_{1}^{2}$. The continuum distribution of charge density also allows a Bolognesi bag by this criterion, as the following argument shows. Suppose the bag has thickness $l$, small compared with $R_{\text {in }}$, so the bag lies between $R_{\text {in }}$ and $R_{\text {in }}+l$. The charge in the bag is $q_{0} l$ and the bag radius is $R_{\text {in }}$. The expected slope of the Higgs field just outside the bag is $q_{0} l / R_{\mathrm{in}}^{2}$. But this agrees with the slope (24). So a Bolognesi bag is present, but this calculation doesn't determine its thickness, nor its charge. However there is a further consideration, as we have specified the charge density throughout the monopole galaxy, and in particular, near the inner radius. The volume per unit charge here is $R_{\text {in }}^{2} / q_{0}$ (dropping a $4 \pi$ factor), so the separation of monopoles is

$$
s=\frac{R_{\mathrm{in}}^{2 / 3}}{q_{0}^{1 / 3}} .
$$

If we identify $s$ with $R_{\text {in }} / \sqrt{q_{0}} l$, the separation of monopoles within a Bolognesi bag of radius $R_{\text {in }}$ and charge $q_{0} l$, we deduce that $l=R_{\text {in }}^{2 / 3} / q_{0}^{1 / 3}=s$. The thickness of the bag $l$ is the same as the monopole separation within the bag, which is what we expect. The charge of the bag is $Q_{\mathrm{bag}}=\left(q_{0} R_{\mathrm{in}}\right)^{2 / 3}$. 
So we have now consistently determined the parameters of the Bolognesi bag at the centre of a monopole galaxy. In terms of the total charge $Q$ and the inner and outer radii of the monopole galaxy, the bag charge is

$$
Q_{\text {bag }}=Q^{2 / 3}\left(\frac{R_{\text {in }}}{R_{\text {out }}}\right)^{2 / 3}
$$

using the approximation that $Q=q_{0} R_{\text {out }}$. $Q_{\text {bag }}$ is very much smaller than $Q$, so almost all monopoles form a dilute gas outside the bag. Similarly, the thickness is

$$
l=\frac{R_{\mathrm{in}}^{2 / 3} R_{\mathrm{out}}^{1 / 3}}{Q^{1 / 3}} .
$$

Therefore, a monopole galaxy has a feature rather analogous to the black hole at the centre of a real galaxy, namely an inner magnetic bag where monopoles have coalesced, carrying a small fraction of the total charge. Given the bag radius, and the outer radius and total charge of the monopole galaxy, the charge of the bag is determined. This contrasts with the black hole in a galaxy, whose mass is not theoretically determined.

We can have some fun with the monopole arithmetic. Suppose a monopole galaxy has charge $10^{12}$, and the ratio of outer to inner radius is $10^{3}$. Then the magnetic bag at the centre has charge $10^{6}$. These numbers resemble the analogous numbers for a real galaxy and its black hole (with charge identified with number of solar masses). So the monopole equations, which forbid $\phi$ to be negative, provide a useful model for regularising the density singularity inside a galaxy, as well as providing an interesting analogy for understanding why central black holes occur in galaxies.

\section{Spectral Radii of Monopoles}

This section depends on the theory of monopole spectral curves, and the details, other than the next paragraph, may be skipped.

There are BPS monopole solutions of charges $N=3,4,5,7$ with the symmetries and shapes of the platonic solids. They provide small scale models of the Bolognesi bag that is expected at large $N$. Here we use the known spectral curve of each of these monopoles to calculate its spectral radius $D_{N}$, which is a precisely defined estimate of the monopole size, and compare with the Bolognesi estimate of the radius, $R_{N}$. We will show them to be similar. We include the $N=1$ and toroidal $N=2$ monopoles in the calculations, 
and also make some remarks about the sequence of axisymmetric, toroidal monopoles that exist for all $N$.

Any BPS monopole is characterised by its spectral curve. This is obtained easily from the Nahm data of the monopole, or equivalently, using the normalisable solutions of the Hitchin equation in the monopole background. For details see, e.g. [1, 2]. The spectral curve is a complex curve lying in the space of all straight lines in $\mathbb{R}^{3}$ (which is the mini-twistor space $T P^{1}$, a non-compact complex surface). The complex coordinates of $T P^{1}$ are $\xi$ parametrising the direction of a straight line (obtained by usual stereographic projection), and $\eta$ which parametrises the intersection point of the line with a (complex) plane orthogonal to the direction $\xi$. Given a line with coordinates $\xi$ and $\eta$, the points $\left(x_{1}, x_{2}, x_{3}\right) \in \mathbb{R}^{3}$ that lie on it are those satisfying

$$
\eta-\left(x_{2}-i x_{1}\right)+2 x_{3} \xi+\left(x_{2}+i x_{1}\right) \xi^{2}=0 .
$$

Important for us is to note that the distance between the line and the origin in $\mathbb{R}^{3}$ (the distance to the closest point on the line) is

$$
d=\frac{|\eta|}{1+|\xi|^{2}} .
$$

If $\eta=0$ then the line passes through the origin.

The spectral curve of a given monopole of charge $N$ is an algebraic curve in $T P^{1}$ of the form

$$
P_{N}(\eta, \xi)=0
$$

where $P_{N}$ is a polynomial of degree $N$ in $\eta$ and (generically) of degree $2 N$ in $\xi$. For fixed direction $\xi$, there are $N$ solutions $\eta$. So the monopole has $N$ straight lines associated with it in this direction (and in every other direction). These are called spectral lines. Heuristically, spectral lines pass through regions where the energy density of the monopole is concentrated and the fields are fully non-abelian. In particular, for $N$ well separated unit charge monopoles, these lines pass through all the monopole cores.

The spectral curve is compact. This implies that the distance $d$ defined above has a finite maximum value $D$ as $\xi$ and $\eta$ vary over the spectral curve. Let us call $D$ the spectral radius of the monopole. There is a notion of a centred monopole (with centre of mass at the origin). The spectral radius is most useful when applied to a centred monopole, and is invariant under rotations of the monopole.

Let us now evaluate the spectral radii of the most symmetric, centred monopoles of low charge. We consider the basic $N=1, O(3)$-symmetric monopole, the toroidal $N=2$ monopole, and the bag-like monopoles of charges $N=3,4,5,7$ with tetrahedral, cubic, octahedral and dodecahedral 
form [7, 17, 18]. There is also a monopole of charge $N=11$ with icosahedral form [19]. They are unique for these charges, and no monopoles of smaller charge have these platonic symmetries.

The spectral curves of all these have been determined, except the $N=11$ example. They are, respectively 2 ,

$$
\begin{aligned}
\eta & =0, \\
\eta^{2}+\frac{\pi^{2}}{4} \xi^{2} & =0, \\
\eta^{3}+\frac{\Gamma\left(\frac{1}{6}\right)^{3} \Gamma\left(\frac{1}{3}\right)^{3}}{48 \sqrt{3} \pi^{3 / 2}} i \xi\left(\xi^{4}-1\right) & =0, \\
\eta^{4}+\frac{3 \Gamma\left(\frac{1}{4}\right)^{8}}{1024 \pi^{2}}\left(\xi^{8}+14 \xi^{4}+1\right) & =0, \\
\eta^{5}+\frac{3 \Gamma\left(\frac{1}{4}\right)^{8}}{256 \pi^{2}}\left(\xi^{8}+14 \xi^{4}+1\right) \eta & =0, \\
\eta^{7}+\frac{\Gamma\left(\frac{1}{6}\right)^{6} \Gamma\left(\frac{1}{3}\right)^{6}}{64 \pi^{3}} \xi\left(\xi^{10}+11 \xi^{5}-1\right) \eta & =0 .
\end{aligned}
$$

Use of the identity $\Gamma(1 / 6)=(3 / \pi)^{1 / 2} 2^{-1 / 3}(\Gamma(1 / 3))^{2}$ gives alternative expressions for the constants above [21]. In the cases $N>2$, the polynomial $\mathcal{K}(\xi)$ that appears here is a Klein polynomial associated with the relevant platonic solid [22]. This is a polynomial that vanishes in the directions of all the face centres, or all the edge centres, or all the vertices of the solid. Note that we are following the conventions of [2] in this section, where the asymptotic behaviour of the Higgs field is $\phi=1-N / 2 r$. The Bolognesi radius is therefore $R_{N}=N / 2$.

For given $\xi$ it is easy to find the roots $\eta$ for each of these equations. One of the roots is $\eta=0$ in cases (35) and (36). The non-zero roots all have the same magnitude $|\eta|_{\xi}$. Evaluating $d_{\xi}=|\eta|_{\xi} /\left(1+|\xi|^{2}\right)$ therefore gives the maximum distance from the origin of spectral lines in the direction $\xi . d_{\xi}$ is a function with the symmetry of the monopole. The spectral radius, which we denote by $D_{N}$ for these special monopoles, is the maximum value of $d_{\xi}$ over all values of $\xi$.

For $N=1, d_{\xi}=0$ for all $\xi$, so $D_{1}=0$. For $N=2$

$$
d_{\xi}=\frac{\pi}{2} \frac{|\xi|}{1+|\xi|^{2}}
$$

\footnotetext{
${ }^{2}$ Included in equations (34) and (35) is a factor 16 correction to equations in [7, 18, see [20].
} 
and this is maximal on the equator $|\xi|=1$. So

$$
D_{2}=\frac{\pi}{4} \simeq 0.785 \text {. }
$$

For higher $N$ it is not always easy to find $D_{N}$ by elementary calculus. A reasonable conjecture is that it occurs in a direction with a simple meaning for the relevant platonic solid. Let $\mathrm{V}, \mathrm{E}$ and $\mathrm{F}$ denote the sets of directions $\xi$ corresponding to the vertices, edges and faces of the solid. Let $n_{\mathrm{V}}, n_{\mathrm{E}}$ and $n_{\mathrm{F}}$ denote the number of these elements. By Euler's theorem, $n_{\mathrm{V}}-n_{\mathrm{E}}+n_{\mathrm{F}}=2$. Because $|\eta|_{\xi}$ has no branch crossing as $\xi$ varies, $d_{\xi}$ is a smooth Morse function on the 2-sphere. Denote the number of its local maxima, saddle points and minima by $n_{\max }, n_{\text {saddle }}$ and $n_{\min }$. By the Morse relations, $n_{\max }-n_{\text {saddle }}+$ $n_{\min }=2$. For a simple function with the symmetry of the platonic solid, two possible arrangements of the stationary points are therefore that the maxima occur at $\mathrm{V}$ (or $\mathrm{F}$ ), the saddle points at $\mathrm{E}$ and the minima at $\mathrm{F}$ (or $\mathrm{V})$. Exchanging $\mathrm{V}$ and $\mathrm{F}$ means replacing the solid by its dual.

We will assume that for the spectral curves above, $d_{\xi}$ is such a simple Morse function. In each case the minimum value of $d_{\xi}$ is zero, the minima occuring at the roots of the Klein polynomial $\mathcal{K}(\xi)$ appearing in the spectral curve equation. For $N=4, \mathcal{K}(\xi)=\xi^{8}+14 \xi^{4}+1$ is the vertex polynomial of the cube, so the minima are at the vertices, and the maxima at the face centres of the cube. For $N=5, \mathcal{K}(\xi)$ is the same, so the conclusion is the same (although we can now say the minima are on the faces of an octahedron and the maxima at the vertices). For $N=7, \mathcal{K}(\xi)=\xi\left(\xi^{10}+11 \xi^{5}-1\right)$ is the face polynomial of the dodecahedron (with one root at infinity), so the maxima of $d_{\xi}$ are at the vertices of the dodecahedron.

The case $N=3$ is more tricky. While the monopole itself has tetrahedral symmetry, and is not invariant under an inversion, the function $d_{\xi}$ has this inversion symmetry, because if a line in $\mathbb{R}^{3}$ belongs to the spectral curve, then so does the line obtained by reversing its direction. Inversion sends $\xi$ to its antipode $-1 / \bar{\xi}$. The distances $d$ for this pair are the same. The function $d_{\xi}$ therefore has not just tetrahedral symmetry, but cubic symmetry. For $N=3, \mathcal{K}(\xi)=\xi\left(\xi^{4}-1\right)$ is the edge polynomial of the tetrahedron, so the edge centres are where $d_{\xi}$ has its minima. However, these points are also the face centres of the cube whose vertices combine those of the tetrahedron and its dual. The maxima of $d_{\xi}$ occur on the vertices of this cube, that is, on both the faces and vertices of the initial tetrahedron. (The 12 saddle points are at the edge centres of the cube, and are distributed three per face of the tetrahedron.) Having identified the locations of the maxima of $d_{\xi}$ for each of the platonic monopoles we can now calculate their spectral radii. 
For $N=3$, one vertex of the cube just described is at

$$
\xi=\frac{1+i}{2}(\sqrt{3}-1)
$$

this being a root of the Klein polynomial $\xi^{8}+14 \xi^{4}+1$. Solving for $|\eta|_{\xi}$ from the $N=3$ spectral curve gives

$$
d_{\xi}=\frac{\Gamma\left(\frac{1}{6}\right) \Gamma\left(\frac{1}{3}\right)}{2^{4 / 3}(3 \pi)^{1 / 2}} \frac{\left|\xi\left(\xi^{4}-1\right)\right|^{1 / 3}}{1+|\xi|^{2}},
$$

and evaluating this with the above value of $\xi$, we find

$$
D_{3}=\frac{\Gamma\left(\frac{1}{6}\right) \Gamma\left(\frac{1}{3}\right)}{2^{4 / 3}(3 \pi)^{1 / 2}} \frac{2^{1 / 6}}{3^{1 / 2}} \simeq 1.249 .
$$

It is easy to verify directly that this is the maximum value of $d_{\xi}$. Firstly, for given $|\xi|, \xi^{4}$ should be negative, which determines the possible arguments of $\xi$, and then the value of $|\xi|$ which maximises the remaining real expression can be found by differentiation.

For $N=4$

$$
d_{\xi}=\left(\frac{3 \Gamma\left(\frac{1}{4}\right)^{8}}{1024 \pi^{2}}\right)^{1 / 4} \frac{\left|\xi^{8}+14 \xi^{4}+1\right|^{1 / 4}}{1+|\xi|^{2}} .
$$

The maxima of $d_{\xi}$ are at the face centres of the cube, the roots of $\xi\left(\xi^{4}-1\right)$, one of which is at $\xi=0$. Hence

$$
D_{4}=\left(\frac{3 \Gamma\left(\frac{1}{4}\right)^{8}}{1024 \pi^{2}}\right)^{1 / 4} \simeq 1.725 .
$$

One can verify that $d_{\xi}$ has the same value at other face centres, e.g. at $\xi=1$, and it has a lower value at the edge centres, e.g. at $\xi=\sqrt{2}-1$. Again, $D_{4}$ can be directly verified to be the maximum value of $d_{\xi}$. The maximum occurs for $\xi^{4}$ real and non-negative, so we can choose $\xi$ real. Then we can find the stationary points of $\left(\xi^{8}+14 \xi^{4}+1\right)^{1 / 4} /\left(1+\xi^{2}\right)$ by differentiation, and identify the maxima at $\xi=0, \pm 1$ (and $\xi=\infty$ ).

For $N=5$ the geometry is the same, but the spectral curve equation has an extra factor of 4 (and an additional overall factor of $\eta$ ). This means that the non-zero roots $\eta$ are multiplied by $\sqrt{2}$, so $D_{5}=\sqrt{2} D_{4}$. Therefore

$$
D_{5} \simeq \sqrt{2} \times 1.725 \simeq 2.440 .
$$

For $N=7$ we need a vertex of the dodecahedron, a root of the Klein polynomial $\xi^{20}-228 \xi^{15}+494 \xi^{10}+228 \xi^{5}+1$. We have found numerically 
that one of the roots is $\xi=-0.3382612$ with $\xi^{5}=-0.00442854$ [23]. Solving the spectral curve equation gives

$$
d_{\xi}=\frac{\Gamma\left(\frac{1}{6}\right) \Gamma\left(\frac{1}{3}\right)}{2 \pi^{1 / 2}} \frac{\left|\xi\left(\xi^{10}+11 \xi^{5}-1\right)\right|^{1 / 6}}{1+|\xi|^{2}},
$$

and evaluating this for the numerically obtained $\xi$ gives

$$
D_{7} \simeq 3.176 \text {. }
$$

Here we have not shown directly that this is the maximum value of $d_{\xi}$.

Our combined results are that for $N=1,2,3,4,5,7$, the values of the spectral radius $D_{N}$ are $0,0.785,1.249,1.725,2.440,3.176$. For comparison the Bolognesi radius is $R_{N}=N / 2$, with values $0.5,1.0,1.5,2.0,2.5,3.5$. The ratios $D_{N} / R_{N}$ are $0,0.785,0.833,0.863,0.976,0.907$, and these appear to be approaching 1 as $N$ increases. These calculations therefore confirm that the platonic monopoles are models for the Bolognesi magnetic bag at small charge.

Finally, we consider the spectral radii of the sequence of toroidal monopoles that exist for all $N$. Using the spectral curves obtained by Hitchin [10], one sees that the largest roots $\eta$ for any $\xi$ are

$$
\eta= \pm \frac{N-1}{2} \pi \xi
$$

so $|\eta|_{\xi}=(N-1) \pi|\xi| / 2$. It follows that the maximum value of $d_{\xi}=|\eta|_{\xi} /(1+$ $\left.|\xi|^{2}\right)$ is $(N-1) \pi / 4$, and this is the spectral radius of the charge $N$ toroidal monopole. Bolognesi has estimated this using an abelian magnetic disc approximation, finding a radius $N \pi / 4$ (rescaling by 2 , as elsewhere in this section) [11. These radii agree asymptotically, the spectral radius being slightly smaller as for the platonic monopoles.

We see that for any $N$, the toroidal monopole in the magnetic disc approximation is larger than the spherical magnetic bag by a factor $\pi / 2$, which is further evidence that the spherical magnetic bag is the most compact monopole. We can also verify that for $N=3,4,5,7$ the spectral radius of the toroidal monopole is larger than that of the platonic monopole.

\section{Conclusions}

Magnetic bags are clearly an important type of BPS monopole solution, even if their existence has not been rigorously established. The approximately 
spherical magnetic bag, for each large charge $N$, is probably the most compact monopole within the whole $4 N$-dimensional moduli space of solutions, and we have found new evidence for this.

A large class of new monopole solutions has been found, using the same abelian approximation that gave the magnetic bag solution. These are spherical clusters of monopoles, and they are all larger than the Bolognesi bag of the same charge. Some of these clusters have bag structures in their interior, and it would be interesting to investigate in more detail how the bag joins on to the gas of more isolated monopoles outside the bag.

We have shown here that the known platonic monopoles with $N=$ 3, 4,5,7 form a sequence that look bag-like, and that with increasing $N$ their spectral radii approach the asymptotic value of the radius predicted for a magnetic bag, $R=N$. There should be further, exact, bag-like solutions with platonic symmetry. The first of these has $N=11$. There is then a sequence of rational maps, with icosahedral symmetry and various degrees from 17 up to 97 and beyond, which have been used to construct bag-like Skyrmions [24]. These could produce bag-like monopoles too, if one interpreted these rational maps as the Jarvis maps of monopoles, and implemented the monopole construction of Ioannidou and Sutcliffe [25]. Less clear is the characterisation of the Jarvis maps which correspond to the spherical monopole clusters of variable density that we have discussed. A spherical cluster would probably arise from a Jarvis map whose zeros and poles were distributed randomly but evenly over $S^{2}$. However, it is difficult to construct a monopole of large charge from a rational map of large degree, as it is still necessary to solve a partial differential equation numerically.

We have interpreted the monopole cluster solutions found here in astrophysical terms. This was motivated by the apparently good analogy between a magnetic bag of charge $N$ and a black hole of mass $M$ [14]. The bag radius is $R=N$ and (in units where Newton's constant and the speed of light are 1 ) the horizon radius of the black hole is $R=2 M$.

For the monopole clusters, we have seen that $\phi$ runs either from 0 at the centre to 1 at infinity, or from $c$ at the centre to 1 at infinity, with $c>0$. Moreover, if $\phi=0$ at the centre, then there is a Bolognesi bag there. These possibilities are analogous to the result that the Newtonian gravitational potential of an astrophysical cluster (in our units) runs from some negative value no less than $-1 / 2$ at the centre to 0 at infinity, and if the central value is $-1 / 2$, then light cannot escape, and there is a central black hole. We have seen that the objects we call monopole planets have $c>0$ at the centre, so these are like real planets or stars that have no black hole inside. The monopole galaxies, on the other hand, can have $c=0$. The inner part of such a monopole galaxy is a magnetic bag, and this is analogous to a black 
hole at the centre of a real galaxy.

The conclusion seems to be that clusters of BPS monopoles of large magnetic charge provide a new, non-relativistic analogy for both black holes and less dense astrophysical objects. The analogy does, of course, have limitations. In particular, the monopole solutions considered here are all static, whereas clusters of stars could only be approximately stationary if the individual objects in the cluster were orbiting the centre. Also, unfortunately, monopoles are not physically observable, although they can be studied mathematically, and numerical solutions presented visually [2].

In summary, the analogy rests on three points. First, both BPS monopoles and astrophysical objects can be approximately described by an abelianized scalar potential $\phi$ satisfying a Poisson equation. In the monopole case $\phi$ is the abelianized Higgs field; in the astrophysical case it is the Newtonian gravitational potential in natural units. In both cases, $\phi$ has to be course-grained, to smooth out the sharp dips due to unit charge monopoles in monopole clusters, or stars within galaxies. Second, the potential $\phi$ cannot be less than a critical value: 0 in the monopole clusters, and $-1 / 2$ in astrophysical objects. In the monopole case this is not so much because the true Higgs field $\Phi$ has minimum magnitude zero at the centre of monopoles, but rather because $\phi$ determines the local size of a unit charge monopole, which has to remain finite; in the gravitational case it is because the Newtonian approximation breaks down where $\phi$ reaches $-1 / 2$, as light cannot escape from such a region, and the full machinery of Einstein's equations is needed. Third, the region where $\phi$ has its critical value is a region of very compact matter, and exhibits an exotic size scaling, with its radius rather than volume proportional to the amount of matter. For monopoles, such a region is the interior of a Bolognesi magnetic bag, the most compact arrangement of magnetic charge. The radius of the bag is equal to the bag's magnetic charge. For gravitating matter, the region is the horizon of a black hole (with the Newtonian picture invalid inside), whose radius is twice the black hole's mass.

\section{Acknowledgements}

This research was largely carried out at the Simons Center for Geometry and Physics, Stony Brook University. I wish to thank Sir Michael Atiyah and the SCGP staff for their invitation and hospitality. I also thank Nigel Hitchin and Sergei Cherkis for discussions. 


\section{References}

[1] M. F. Atiyah and N. J. Hitchin, The Geometry and Dynamics of Magnetic Monopoles, Princeton University Press, 1988.

[2] N. Manton and P. Sutcliffe, Topological Solitons, Cambridge University Press, 2004.

[3] Ya. Shnir, Magnetic Monopoles, Berlin Heidelberg, Springer, 2005.

[4] A. Jaffe and C. Taubes, Vortices and Monopoles, Boston, Birkhäuser, 1980.

[5] N. S. Manton, The force between 't Hooft-Polyakov monopoles, Nucl. Phys. B126, 525 (1977).

[6] S. Jarvis, A rational map for Euclidean monopoles via radial scattering, J. reine angew. Math. 524, 17 (2000).

[7] N. J. Hitchin, N. S. Manton and M. K. Murray, Symmetric monopoles, Nonlinearity 8, 661 (1995).

[8] P. Forgács, Z. Horváth and L. Palla, Exact multimonopole solutions in the Bogomolny-Prasad-Sommerfield limit, Phys. Lett. B99, 232 (1981).

[9] M. K. Prasad and P. Rossi, Construction of exact Yang-Mills-Higgs multimonopoles of arbitrary charge, Phys. Rev. Lett. 46, 806 (1981).

[10] N. J. Hitchin, On the construction of monopoles, Commun. Math. Phys. 89, 145 (1983).

[11] S. Bolognesi, Multi-monopoles and magnetic bags, Nucl. Phys. B752, 93 (2006).

[12] K.-M. Lee and E. J. Weinberg, BPS magnetic monopole bags, Phys. Rev. D79, 025013 (2009).

[13] J. Binney and S. Tremaine, Galactic Dynamics (2nd ed.), Princeton University Press, 2008.

[14] S. Bolognesi, Magnetic bags and black holes, Nucl. Phys. B845, 324 (2011).

[15] R. S. Ward, Monopole wall, Phys. Rev. D75, 021701(R) (2007).

[16] D. Harland, The large $N$ limit of the Nahm transform, Commun. Math. Phys. published online (2011).

[17] C. J. Houghton and P. M. Sutcliffe, Tetrahedral and cubic monopoles, Commun. Math. Phys. 180, 343 (1996).

[18] C. J. Houghton and P. M. Sutcliffe, Octahedral and dodecahedral monopoles, Nonlinearity 9, 385 (1996). 
[19] C. J. Houghton, N. S. Manton and P. M. Sutcliffe, Rational maps, monopoles and Skyrmions, Nucl. Phys. B510, 507 (1998).

[20] P. M. Sutcliffe, BPS monopoles, Int. J. Mod. Phys. A12, 4663 (1997).

[21] C. J. Houghton, N. S. Manton and N. M. Romão, On the constraints defining BPS monopoles, Commun. Math. Phys. 212, 219 (2000).

[22] F. Klein, Lectures on the Icosahedron, London, Kegan Paul, 1913.

[23] http://www.hvks.com/Numerical/websolver.php

[24] R. A. Battye, C. J. Houghton and P. M. Sutcliffe, Icosahedral Skyrmions, J. Math. Phys. 44, 3543 (2003).

[25] T. Ioannidou and P. M. Sutcliffe, Monopoles from rational maps, Phys. Lett. B457, 133 (1999). 